\title{
Elastic Modulus Calculation Model of a Soil-Rock Mixture at Normal or Freezing Temperature Based on Micromechanics Approach
}

\author{
Hao Yang, ${ }^{1,2}$ Zhong Zhou, ${ }^{1,2}$ Xiangcan Wang, ${ }^{1,2}$ and Qifang Zhang ${ }^{1,2}$ \\ ${ }^{1}$ School of Civil Engineering of Central South University, Changsha 410075, China \\ ${ }^{2}$ National Engineering Laboratory for High Speed Railway Construction, Changsha 410075, China \\ Correspondence should be addressed to Zhong Zhou; 369144091@qq.com
}

Received 11 December 2014; Revised 13 February 2015; Accepted 15 February 2015

Academic Editor: Pavel Lejcek

Copyright (C) 2015 Hao Yang et al. This is an open access article distributed under the Creative Commons Attribution License, which permits unrestricted use, distribution, and reproduction in any medium, provided the original work is properly cited.

\begin{abstract}
Considering rock wrapped by soil in the mesoscopic structure of soil-rock mixture at normal temperature, a two-layer embedded model of single inclusion composite material was established to obtain the elastic modulus of soil-rock mixture. Given an interface ice interlayer attached between the soil and rock interface in the mesoscopic structure of soil-rock mixture at freezing temperature, a three-layer embedded model of double inclusion composite material and multistep multiphase micromechanics model was established to obtain the elastic modulus of a frozen soil-rock mixture. With the effect of structure pore with soil-rock mixture at normal temperature taken into consideration, its elastic modulus was calculated with the three-layer embedded model. An experimental comparison found that the predicted effect of the three-layer embedded model on the soil-rock mixture was better than that of the two-layer embedded model. The elastic modulus of soil-rock mixture gradually increased with the increase in rock content regardless of temperature. The increase rate of the elastic modulus of the soil-rock mixture increased quickly especially when the rock content is between $50 \%$ and $70 \%$. The elastic modulus of the frozen soil-rock mixture is close to four times higher than that of the soil-rock mixture at a normal temperature.
\end{abstract}

\section{Introduction}

Soil-rock mixture is an important engineering composite material that is widely distributed and abundant in nature and is suitable as local engineering materials. This material has the advantages of good resistance to compression performance, high shearing strength, strong bearing capacity, and good permeability. Moreover, it is widely used in dams, artificial foundations, storage yard of ports, flood control levees, roadbed, and engineering works for comprehensive treatment of urban river banks [1].

Elasticity modulus is a key material parameter [2]. It can be applied to calculate landslide mechanism and design support form of soil-rock mixture slope in slope-supported and underground engineering. It also can be used to design roadbed padding and predict the subgrade under load mechanical response of soil-rock mixture roadbed in subgrade engineering. The main methods of determining the elastic modulus of a soil-rock mixture project are test methods and empirical formula methods. Test methods use indoor or in situ test to determine the elastic modulus of soilrock mixture. However, the indoor test method is time consuming and can only determine elastic modulus after a soilrock mixture has been made. In situ tests are expensive and may not achieve the desired test accuracy because of problems with the testing equipment. The scope of various empirical formula methods is conditional. If the actual condition is different from that of the empirical formula method, the predicted results by the empirical formula method may show errors. In fact, both test and empirical formula methods based on the macro level did not reflect the mechanical properties at microscopic scales [3]. Therefore, establishing an appropriate micromechanical model is necessary to accurately predict the elastic modulus of soil-rock mixture at the design stage to achieve effective design applications in slope retaining and roadbed padding engineering. Moreover, soil-rock mixture 


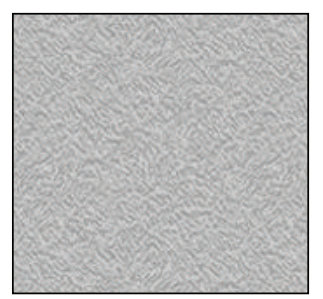

(a) $C_{\mathrm{R}}=0 \%$

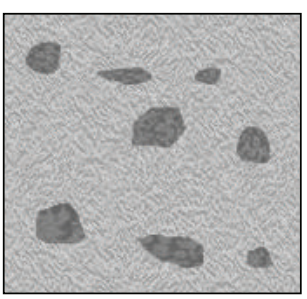

(b) $0 \%<C_{R}<25 \%$

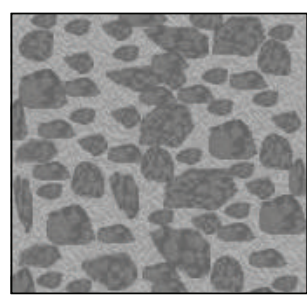

(c) $25 \%<C_{R}<75 \%$

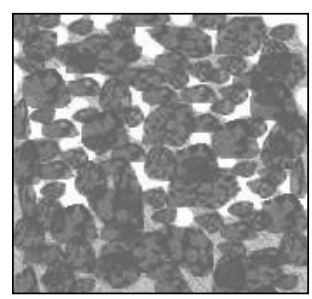

(d) $75 \%<C_{\mathrm{R}}<100 \%$

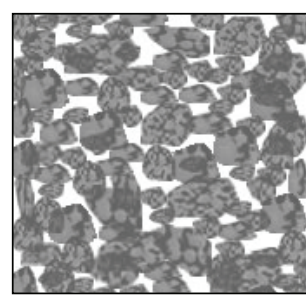

(e) $C_{R}=100 \%$

FIGURE 1: Different rock contents of soil-rock mixture medium macrostructure diagram.

is widely distributed in frozen ground areas throughout the world. With the development of engineering construction in cold regions, research on freeze-thaw action in the soilrock mixture has shifted from an overall study to elaboration of the effect of various physical and mechanical parameters through tests and theoretical analysis [4]. Therefore, studying the elastic modulus of frozen soil-rock mixture in the freezing condition by establishing a suitable micromechanical model is important.

1.1. Definition of Soil-Rock Mixture. A soil-rock mixture refers to an extremely nonuniform, loose system composed of rock blocks (of engineering scales and high strength), fine soil grains, and pores [5]. Medley [6] coined the term bimsoils or bimrocks (block-in-matrix soils or rocks) to refer to rock and soil medium composed of fine-grained soil (or the agglutinating mixture matrix) with an embedded important project block. The Dictionary of Geology and Mineralogy refers to this mixed rock and soil, which contain different particle sizes of itself or foreign debris and matrix mud embedded with rock, as mélange [7].

A soil-rock mixture is composed of coarse grains (broken rock block, gravel) and fine grains (sandy soil, clay) with a wide range of grain sizes. According to the normal specification of an engineering society (the discussion is in Section 4), $5 \mathrm{~mm}$ is used as a threshold to differentiate coarse and fine grains such that those with grain size $<5 \mathrm{~mm}$ are defined as fine grains (soil), whereas those with grain size $>5 \mathrm{~mm}$ are coarse grain (broken rock). The relative volumetric percentage of coarse grains is called rock content, denoted as $C_{R}$. Figure 1 illustrates the macrostructure of the soil-rock mixture with variable rock content. (1) Coarse broken rocks are suspended in fine soil grains when $C_{\mathrm{R}}$ is not more than 25\% (Figures 1(a) and 1(b)); the electrical resistivity of the mixture is essentially determined by the soil body. (2) Broken rocks constitute the skeleton with soils filling the pores while $C_{\mathrm{R}}$ is between $25 \%$ and $75 \%$ (Figure $1(\mathrm{c})$ ). Under such condition, the electrical resistivity of the mixture is determined together by the soil and rock. Thus, the mixture is called soil-rock mixture, while $C_{\mathrm{R}}$ is between $25 \%$ and $75 \%$. (3) A tight and close skeleton is formed between the broken rocks, which prevented complete compactness of the pores of the soil skeleton while the $C_{\mathrm{R}}$ is higher than $75 \%$ (Figures 1(d) and $1(\mathrm{e})$ ). Under such a condition, the electrical resistivity of the mixture is mainly determined by the broken rocks, and the structure is called broken rock body [8].
1.2. Elastic Modulus Prediction of Soil-Rock Mixture. Research on equivalent physical properties prediction of composite began in 1906 [9]. The whole mechanical behavior or mixed material macroscopic mechanical properties was not the same as any component materials; however, they are affected by the formation of various components that commonly play a different role in a complex system $[10,11]$. For mixed materials, this is composed of only two kinds of materials. The representative and most commonly used theoretical models are parallel model (Voigt model) and series model (Reuss model) [12]. However, these models can provide only the upper and lower equivalent modulus of the soil-rock mixture and cannot accurately predict the effective elastic modulus $[12,13]$.

Few studies focus on the static and dynamic properties of soil-rock mixture as computational tools and test conditions improve. Ma et al. [14] derived the equivalent elastic modulus analytical prediction formula of soil-rock mixture theoretically but ignored the effect of Poisson's ratio; hence, the results had a certain bias. Tests of dynamic and static characteristics of soil-rock mixture have received substantially greater research attention. Zhang [15] obtained material strength properties and soil-rock mixture material stress-strain characteristics according to numerous triaxial compression test results. Jiang and Yang [16] used indoor shear creep test in the Chengdu area in China to study the rheological characteristics and long-term strength of soilrock mixture. Jia and Wang [17] and Wang et al. [18] studied the dynamic strength of soil-rock mixture through an indoor dynamic triaxial test. Qin et al. [19] analyzed large grain soil stress-strain characteristics and shear strength under low confining pressure and the water influence on the strength and deformation of large grain soil by using the large-scale triaxial test. Nevertheless, the experimental study is costly and time consuming and has a certain particularity. Therefore, establishing a general rule is difficult. Furthermore, the study on elastic modulus of frozen soil-rock mixture is only in the preliminary exploration stage because the microstructure of soil-rock mixture at a freezing temperature is more complicated than it is at a normal temperature.

Therefore, studying the mechanical behavior of soilrock mixture at normal or freezing temperature condition should be considered when using the theory and method of the composite material mechanics from the perspective of micromechanics. In this study, soil-rock mixture at normal temperature is regarded as a two-layer embedded composite 


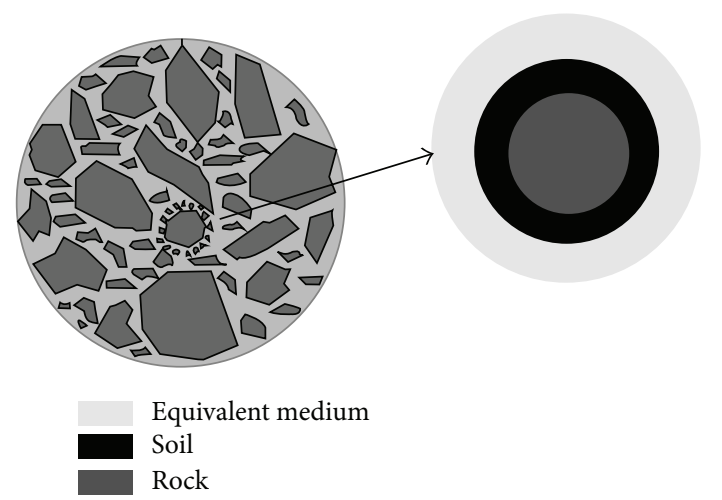

Figure 2: Two-layer embedded model and microstructure sectional drawing of soil-rock mixture.

material that consists of rock particles wrapped by soil matrix embedded in the equivalent medium composite material (see Figure 2). The elastic modulus of the single inclusion composite material is determined assuming that inclusion phase is homogeneous and isotropic and by combining the elastic modulus expression given by $\mathrm{Li}$ et al. [20] with the single shear modulus expression from Timoshenko and Goodier [21]. Thus, using the method of the current study can quickly predict the elastic properties of soil-rock mixture at normal temperature.

From the perspective of micromechanics, a nice interlayer interface is found between soil and rock in the mesoscopic structure of the soil-rock mixture at freezing temperature. Thus, the frozen soil-rock mixture, as a three-layer embedded composite material that consists of ice-rock particles, is wrapped by frozen soil matrix embedded in the equivalent medium composite material (see Figure 6). Therefore, the elastic modulus of multiphase composite can be further determined by using multistep homogenization. The ice-rock grain is regarded as a two-layer embedded composite material that consists of rock particles wrapped by ice matrix and is embedded in the equivalent medium composite material. Using the two-layer embedded composite material elasticity modulus model twice on the frozen soil-rock mixture that is composed of a variety of different sizes and content of rock, ice, and permafrost of multiphase composite material can quickly and accurately predict the elastic properties of frozen soil-rock mixture.

\section{Mesoscopic Model of Soil-Rock Mixture}

Considering a rock wrapped by soil in the mesoscopic structure of soil-rock mixture at normal temperature, a twolayer embedded model of single inclusion composite material was established to obtain the elastic modulus of the soil-rock mixture.

2.1. Two-Layer Embedded Model of Single Inclusion Composite Material. First, a two-layer embedded model is established considering inclusion composite. The inclusion of each particle is seen as the circular inclusion wrapped by a certain

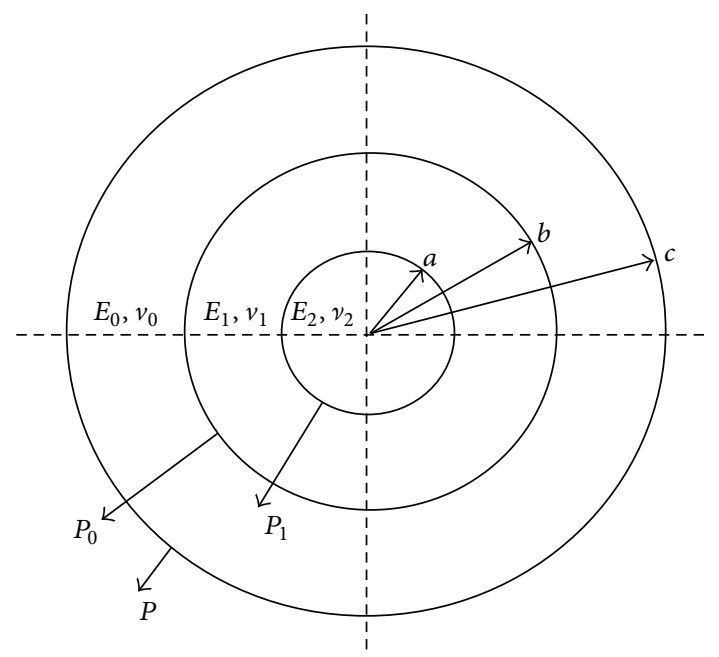

FIGURE 3: Microstructure model of elastic parameter calculation.

thickness of the substrate, which is embedded in an infinite equivalent composite medium, as shown in Figure 2.

Based on the above micromechanical model, Li et al. [20] derived the following analytical solution of elasticity according to classical elastic theory [21] by applying uniform radial stress $P, P_{0}$, and $P_{1}$ (see Figure 3 ) on the boundary of $r=c, r=b$, and $r=a$ :

$$
\begin{aligned}
& u_{0 c}=\frac{1}{E_{0}} \cdot\left[\left(1+v_{0}\right) \frac{b^{2} c^{2}\left(P-P_{0}\right)}{\left(c^{2}-b^{2}\right) c}+\left(1-v_{0}\right) \frac{P c^{2}-P_{0} b^{2}}{c^{2}-b^{2}} c\right], \\
& u_{0 b}=\frac{1}{E_{0}} \cdot\left[\left(1+v_{0}\right) \frac{b^{2} c^{2}\left(P-P_{0}\right)}{\left(c^{2}-b^{2}\right) b}+\left(1-v_{0}\right) \frac{P c^{2}-P_{0} b^{2}}{c^{2}-b^{2}} b\right], \\
& u_{1 b}=\frac{1}{E_{1}} \cdot\left[\left(1+v_{1}\right) \frac{a^{2} b^{2}\left(P_{0}-P_{1}\right)}{\left(b^{2}-a^{2}\right) b}+\left(1-v_{1}\right) \frac{P_{0} b^{2}-P_{1} a^{2}}{b^{2}-a^{2}} b\right], \\
& u_{1 a}=\frac{1}{E_{1}} \cdot\left[\left(1+v_{1}\right) \frac{a^{2} b^{2}\left(P_{0}-P_{1}\right)}{\left(b^{2}-a^{2}\right) a}+\left(1-v_{1}\right) \frac{P_{0} b^{2}-P_{1} a^{2}}{b^{2}-a^{2}} a\right], \\
& u_{2 a}=\frac{1-v_{2}}{E_{2}} p_{1} a,
\end{aligned}
$$

where $u$ is the displacement; $v_{0}$ is Poisson's ratio soil-rock mixture; $v_{1}$ is Poisson's ratio of soil; $v_{2}$ is Poisson's ratio of rock; $E_{0}, E_{1}$, and $E_{2}$ are the elasticity modulus of soil-rock mixture, soil, and rock, respectively; $a$ is the radius of rock; and $b$ is the sum of the thickness of the soil and the radius of rock. According to the deformation compatibility conditions and total strain energy equivalence principle, the effective elastic modulus of composite $E_{0}$ can be calculated as follows:

$$
E_{0}=\frac{E_{1}(1-f)\left(1-v_{0}\right)}{x_{1}-\left(4 E_{2} f / E_{1}(1-f)\left(1-v_{0}\right)+E_{2} x_{2}\right)},
$$


where $E_{1}, E_{2}, v_{1}, v_{2}, a$, and $b$ can be given directly based on the material properties of soil-rock mixture, but $\nu_{0}$ is still unknown:

$$
\begin{aligned}
& x_{1}=f\left(1+v_{1}\right)+\left(1-v_{1}\right), \\
& x_{2}=\left(1+v_{1}\right)+f\left(1-v_{1}\right),
\end{aligned}
$$

where $f$ is the volume ratio of the inclusion. The volume ratio formed by the inclusion and the boundary of the matrix is exactly the volume fraction of composite material. For twodimensional problems, the formula is as follows:

$$
b=\frac{a}{\sqrt{f}} .
$$

The rocks in soil-rock mixture are composed of spheroid shape pebbles and cuboid shape broken rocks. The rocks are random distribution and inlaid each other in threedimensional structure of soil-rock mixture, so the twodimensional structure of soil-rock mixture cross sections are not unique. In this case, the cylindrical fiber micromechanics model is more adapt for the two-dimensional section description of soil-rock mixture.

According to the theoretical formula of single-inclusion composite shear modulus obtained from Christensen and Lo [22] and based on the same two-layer embedded model shown in Figure 3, the effective shear modulus $\mu_{0}$ is calculated as follows to fully determine the effective elastic modulus of composite $E_{0}$ :

$$
\begin{gathered}
{\left[\frac{\mu_{0}}{\mu_{1}}\right]^{2} A+\left[\frac{\mu_{0}}{\mu_{1}}\right] B+D=0,} \\
A=3 f(1-f)^{2}\left(\frac{\mu_{2}}{\mu_{1}}-1\right)\left(\frac{\mu_{2}}{\mu_{1}}+\eta_{2}\right) \\
+\left[\left(\frac{\mu_{2}}{\mu_{1}}\right) \eta_{1}+\eta_{2} \eta_{1}-\left(\left(\frac{\mu_{2}}{\mu_{1}}\right) \eta_{1}-\eta_{2}\right) f^{3}\right] \\
\times\left[f \eta_{1}\left(\frac{\mu_{2}}{\mu_{1}}-1\right)-\left(\left(\frac{\mu_{2}}{\mu_{1}}\right) \eta_{1}+1\right)\right], \\
B=-6 f(1-f)^{2}\left(\frac{\mu_{2}}{\mu_{1}}-1\right)\left(\frac{\mu_{2}}{\mu_{1}}+\eta_{2}\right) \\
+\left[\left(\frac{\mu_{2}}{\mu_{1}}\right) \eta_{1}+\left(\frac{\mu_{2}}{\mu_{1}}-1\right) f+1\right] \\
\times\left[\left(\frac{\mu_{2}}{\mu_{1}}+\eta_{2}\right)\left(\eta_{1}-1\right)-2\left(\left(\frac{\mu_{2}}{\mu_{1}}\right) \eta_{1}-\eta_{2}\right) f^{3}\right] \\
+\left(\eta_{1}+1\right) f\left(\frac{\mu_{2}}{\mu_{1}}-1\right) \\
+\left[\frac{\mu_{2}}{\mu_{1}}+\eta_{2}+\left(\left(\frac{\mu_{2}}{\mu_{1}}\right) \eta_{1}-\eta_{2}\right) f^{3}\right],
\end{gathered}
$$

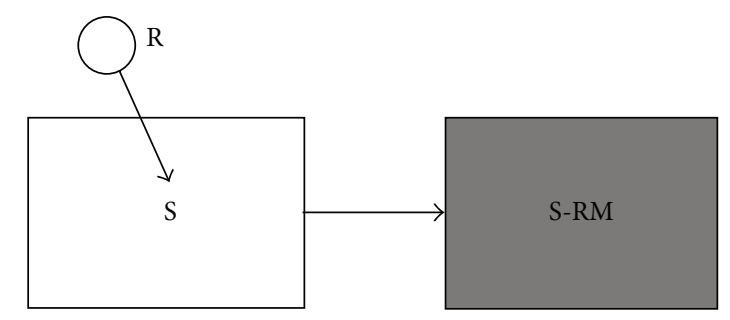

FIGURE 4: Schematic diagram of calculation for single inclusion problem.

$$
\begin{aligned}
D= & 3 f(1-f)^{2}\left(\frac{\mu_{2}}{\mu_{1}}-1\right)\left(\frac{\mu_{2}}{\mu_{1}}+\eta_{2}\right) \\
& +\left[\left(\frac{\mu_{2}}{\mu_{1}}\right) \eta_{1}+\left(\frac{\mu_{2}}{\mu_{1}}-1\right) f+1\right] \\
& \times\left[\frac{\mu_{2}}{\mu_{1}}+\eta_{2}+\left(\left(\frac{\mu_{2}}{\mu_{1}}\right) \eta_{1}-\eta_{2}\right) f^{3}\right], \\
& \eta_{1}=3-4 v_{1}, \quad \eta_{2}=3-4 v_{2},
\end{aligned}
$$

where $\mu_{0}, \mu_{1}$, and $\mu_{2}$ are the shear elasticity modulus of soilrock mixture, the soil, and rock, respectively. Integrating (6a) to (6d) into (5) and according to the relationship between the elastic constants, the shear elasticity modulus of soil-rock mixture can be obtained:

$$
v_{0}=\frac{E_{0}}{2 \mu_{0}}-1 .
$$

Combining (7) and (2), the single composite inclusion elastic modulus $E_{0}$ can be calculated.

2.2. Micromechanics Model of Single Inclusion Composite Material. Soil-rock mixture at normal temperature is a single inclusion composite material. The main idea of calculation is to integrate the rock inclusion (assume its elastic characteristic is R, as determined by the elastic modulus $E$ and Poisson's ratio $v$ material properties, etc.) into the oil matrix material (assume its elastic characteristic is $S$ ), and then use formulas (2) to (7) to complete the homogenization process, thereby obtaining the macroscopic properties of the soil-rock mixture (assume its elastic characteristic is S-RM) at normal temperature. The process is detailed in Figure 4.

When rock inclusion $\mathrm{R}$ is eliminated, the volume fraction ratio of rock inclusion $R$ is

$$
f_{\mathrm{R}}=\frac{V_{\mathrm{R}}}{V_{\mathrm{S}}+V_{\mathrm{R}}},
$$

where $V_{\mathrm{S}}$ is the volume of soil and $V_{\mathrm{R}}$ is the volume of rock.

2.3. Calculation Process of Elastic Modulus of Soil-Rock Mixture. According to micromechanics analysis and combining with the characteristics of the composite materials of soilrock mixture at normal temperature, the calculation process of the elastic modulus of soil-rock mixture at normal temperature is shown in Figure 5. 


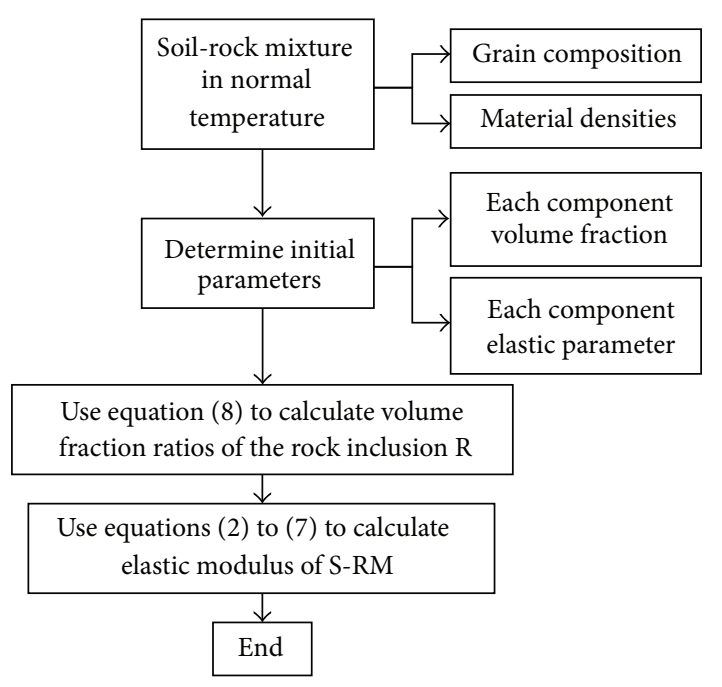

FIGURE 5: Flowchart of the calculation of the elastic modulus of soilrock mixture.

\section{Micromechanics Analysis Model of Soil-Rock Mixture at Freezing Condition}

The pore in soil-rock mixture can be divided into three parts: innate pore in the soil, which is called soil pore; the inherent pore in the rock grain pore, which is called rock pore; and the pore between the soil and rock grain, which is called structural pore. The structural porosity can be calculated according to the inherent porosity of soil-rock mixture, soil, and rock grain. This study considered the soil and rock grain as two entirely independent research objects. Thus, the calculation model needs to consider structural pore as the inclusion for soil-rock mixture.

Rock grain cannot be completely packed by soil, which indicates the loose property of the soil, because of the contact interface pore structure between the soil and the rock grain of soil-rock mixture. The water in the pore structure freezes with reduced temperature. The existence of the pore structure and adsorption on the surface of the rock means that the contact surface also exists in the ice interface between rock grain and soil. Considering the attached ice interface interlayer between soil and rock grain in the mesoscopic structure of soil-rock mixture at freezing temperature, a three-layer embedded model of double inclusion composite material and multistep multiphase micromechanics model were established to obtain the elastic modulus of frozen soilrock mixture.

In Section 2, the influence of contact interface pore structure on the elastic modulus of the soil-rock mixture was not considered in the two-layer embedded model of single inclusion composite material. Accordingly, the three-layer embedded model of double inclusion composite material in Section 3 can be used to recalculate the elastic modulus of the soil-rock mixture at normal temperature to evaluate the predicted effect by comparing the two model calculations with the test data.

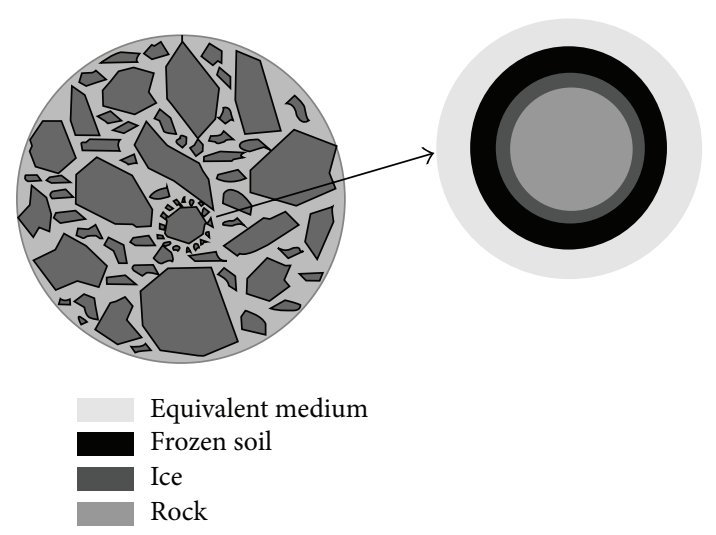

FIgURE 6: Three-layer embedded model and microstructure sectional drawing of frozen soil-rock mixture.

3.1. Three-Layer Embedded Model of Double Inclusion Composite Material. First, the three-layer embedded model is established considering the case of double inclusion composite. The inclusion of each particle is seen as circular inclusion wrapped by a certain thickness of the substrate embedded in an infinite equivalent composite medium. The model is shown in Figure 6.

An analytical solution for the three-phase cylindrical model in piezoelectric composite materials was derived [23, 24]. To simplify the above three-layer embedded model of double inclusion composite material, a mechanical method of breaking the whole into several parts and a gradual solution are used. The rock at the core and the outer wrapped interface are extracted and taken as the research model or single mixed micromechanical model. Its equivalent elastic modulus can be calculated by using the two-layer embedded model of single inclusion composite material, which can be called transition inclusion body. Next, the three-layer embedded model is considered a whole research object. The rock at the core and the outer wrapped ice interface had been converted into a transition inclusion body in a stepby-step process. Therefore, the entire model can be seen as a two-layer embedded model composed of transition inclusion body with frozen soil substrate. Its equivalent elastic modulus parameters, which are the elastic parameters of soil-rock mixture at freezing condition, can be calculated.

\subsection{Micromechanics Model of Multiphase Double Inclusion} Composite Material. Frozen soil-rock mixture is a multiphase double inclusion composite material. A multistep model can be used to handle each kind of inclusion and calculate the elastic modulus of the composite material stepby-step [25].

In the step-by-step process, the rock inclusion (assume its elastic characteristic is R) is first integrated into the ice interface matrix material (assume its elastic characteristic is I, as determined by the elastic modulus $E$ and Poisson's ratio $v$ material properties), and formulas (2) to (7) are used to 


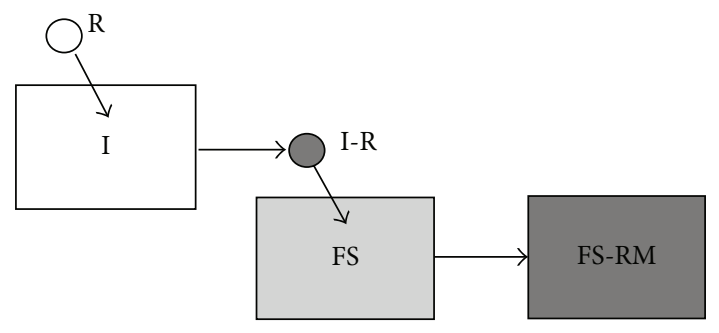

FIGURE 7: Schematic diagram of stepping scheme for multiphase double inclusion problem.

complete the homogenization process and obtain the transition inclusion body (assume its elastic characteristic is I$\mathrm{R})$. Second, the composite material I-R after homogenization is used as a new inclusion, and the new inclusion I-R is integrated into the frozen soil matrix (assume its elastic characteristic is FS). Then, (2) to (7) are used to complete the homogenization process and finally obtain the macroscopic properties (assume its elastic characteristic is FS-RM) of the soil-rock mixture at freezing condition. The process is shown in detail in Figure 7.

When rock inclusion $\mathrm{R}$ is eliminated, the volume fraction ratio of rock inclusion $\mathrm{R}$ is

$$
f_{\mathrm{R}(\mathrm{F})}=\frac{V_{\mathrm{R}}}{V_{\mathrm{I}}+V_{\mathrm{R}}} .
$$

When including the transition of inclusion body I-R, the volume fraction of inclusion body I-R is

$$
f_{\mathrm{I}-\mathrm{R}(\mathrm{F})}=\frac{V_{\mathrm{I}}+V_{\mathrm{R}}}{V_{\mathrm{FS}}+V_{\mathrm{I}}+V_{\mathrm{R}}},
$$

where $V_{\mathrm{I}}$ is the volume of the interface ice, $V_{\mathrm{R}}$ is the volume of rock, and $V_{\mathrm{FS}}$ is the volume of frozen soil.

3.3. Calculation Process of the Elastic Modulus of Frozen SoilRock Mixture. According to the above micromechanics analysis and combined with the characteristics of the composed materials of frozen soil-rock mixture, the calculation process of the elastic modulus of frozen soil-rock mixture can be shown as Figure 8.

\section{Comparison between Model Calculation and Experiment Test}

Knowing the elastic properties and density fraction ratio of soil and rock inclusion body in the soil-rock mixture is necessary when using the proposed method for calculating the elastic modulus of soil-rock mixture. Measuring the rock content ratio and other parameters (the measurements of mixture porosity of soil-rock mixture and the measurements of density with different types of aggregate) ensures that the density fraction ratio of each phase of the asphalt concrete can be calculated, and the elastic modulus of soil-rock mixture can be simply calculated according to the method previously given. The specific calculation process is shown in Figures 5 and 8 .

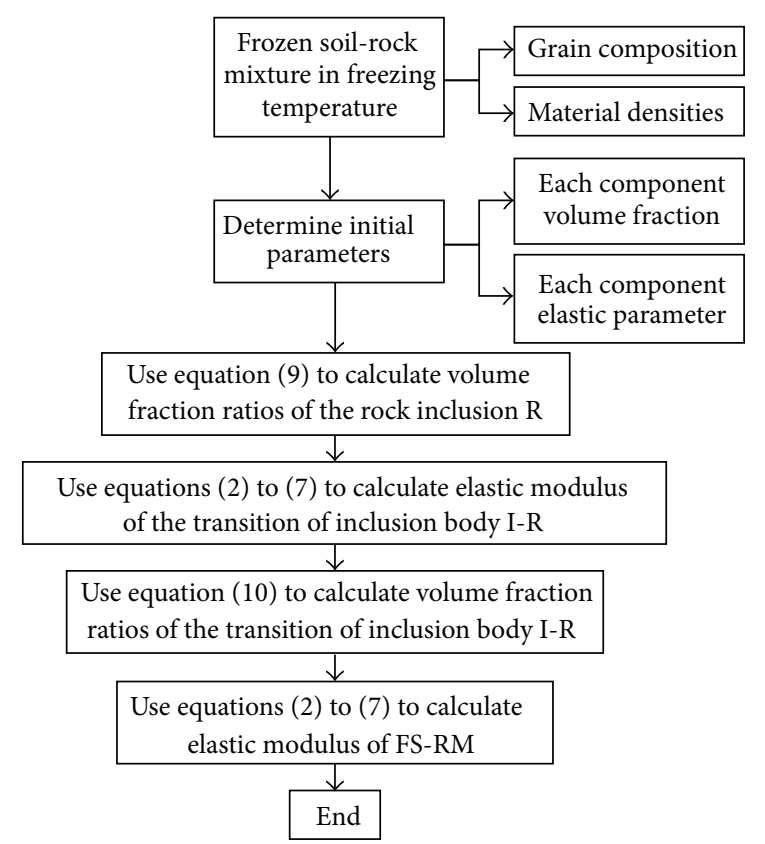

FIgURE 8: Flowchart of the calculation of the elastic modulus of frozen soil-rock mixture.

Guo [26] recommended $5 \mathrm{~mm}$, which is the soil-rock boundary particle size widely used in engineering, as the boundary of sand and gravel in research on classification and naming of soil-rock mixtures. Therefore, the present study used all fine aggregates with a particle size less than $5 \mathrm{~mm}$ as the soil substrate, and coarse aggregates as inclusion are placed one by one in the calculation process. The elastic modulus of soil can be obtained by using TAJ-2000 largescale movement triaxial test instrument for the test platform. However, the test conditions (temperature, loading rate, etc.) used in the test must be fully consistent with the test of soilrock mixture's elastic modulus.

4.1. Volume Fraction Ratios of the Composed Materials. Based on the above two mechanic models, the elastic modulus of the soil-rock mixture is calculated with different rock contents at $20^{\circ} \mathrm{C}$ (referred to as S-RM) and $-20^{\circ} \mathrm{C}$ (referred to as FS-RM). The test samples (the water content of the sample is saturated) are prepared and tested for their modulus of elasticity indoors. The relationship is analyzed, and the difference between the values is calculated by the model and test values [27]. Under the condition of the same rock content, S-RM and FS-RM belong to the same rock gradation samples.

With the use of rock content, measured porosity, and density of each composed material of soil-rock mixture, the volume fraction ratios of the composite material are calculated in each phase. S-RM with five different rock contents in the composed materials and the volume fraction ratios of S-RM are shown in Table 1. For saturated soil-rock mixture with same grading, the volume fraction ratio of interface ice of FS-RM is equal to that of the structure pore of S-RM. 
TABLE 1: Volume fraction ratios of S-RM with different rock contents.

\begin{tabular}{lccc}
\hline \multirow{2}{*}{ Rock content } & \multicolumn{3}{c}{ The volume } \\
& Soil & Roction ratios of S-RM (\%) & Structural pore \\
\hline $30 \%$ & 69.21 & 30.00 & 0.79 \\
$40 \%$ & 58.98 & 40.00 & 1.02 \\
$50 \%$ & 48.75 & 50.00 & 1.25 \\
$60 \%$ & 38.68 & 60.00 & 1.32 \\
$70 \%$ & 28.14 & 70.00 & 1.86 \\
\hline
\end{tabular}

TABLE 2: Elastic modulus of soil and frozen soil.

\begin{tabular}{lcc}
\hline Medium & $\begin{array}{c}\text { Testing temperature } \\
\left({ }^{\circ} \mathrm{C}\right)\end{array}$ & $\begin{array}{c}\text { Elastic modulus } \\
(\mathrm{MPa})\end{array}$ \\
\hline Soil of S-RM & 20 & 2.62 \\
Frozen soil of FS-RM & -20 & 10.15 \\
\hline
\end{tabular}

4.2. Calculation of Elastic Modulus. According to the design of S-RM and FS-RM with five different rock contents, samples are fabricated (the water content of the sample is saturated) with a diameter of $100 \mathrm{~mm}$ and a height of $200 \mathrm{~mm}$. The elastic parameters of soil and frozen soil are measured at 20 and $-20^{\circ} \mathrm{C}$, respectively. The test results are shown in Table 2 .

The rock sample has a diameter of $25 \mathrm{~mm}$ and a height of $50 \mathrm{~mm}$. The elastic parameters are tested by using strain gauge method. The measured data are combined with research findings [28]. The elastic modulus of the rock is taken as $40000 \mathrm{MPa}$, and Poisson's ratio is 0.2 . The elastic modulus of the soil is taken as $2.62 \mathrm{MPa}$, and Poisson's ratio is 0.4 . The elastic modulus of permafrost is taken as $10.15 \mathrm{MPa}$, and Poisson's ratio is 0.38 . The modulus of elasticity of the ice interface is taken as $6820 \mathrm{MPa}$, and Poisson's ratio is 0.3 . The pore structure was formed by an incomplete parcel of soil and rock grain. Therefore, structure pore and soil coexist in the contact surface of the rock grain. The equivalent elastic property of the rock package should be between soil and structure pore water. Thus, this paper can consider the equivalent elastic property as 0.5 times the soil elastic property. The two-layer embedded model of single inclusion composite material was used to calculate the elastic modulus of S-RM. The three-layer embedded model of double inclusion composite material was used to calculate the elastic modulus of S-RM and FS-RM. The calculation results are shown in Table 3.

As seen in Table 3, at freezing temperature, the elastic modulus of the soil-rock mixture is significantly higher than it is at normal temperature. Moreover, the elastic modulus of soil-rock mixture gradually increased with the increase in rock content at both freezing and normal temperature conditions, which is consistent with engineering experience.

4.3. Measurement of Elastic Modulus. To check the elastic modulus of S-RM and FS-RM calculated by the above double inclusion body calculation models, this study measured the elasticity modulus of S-RM and FS-RM with five different rock contents according to the test method below.

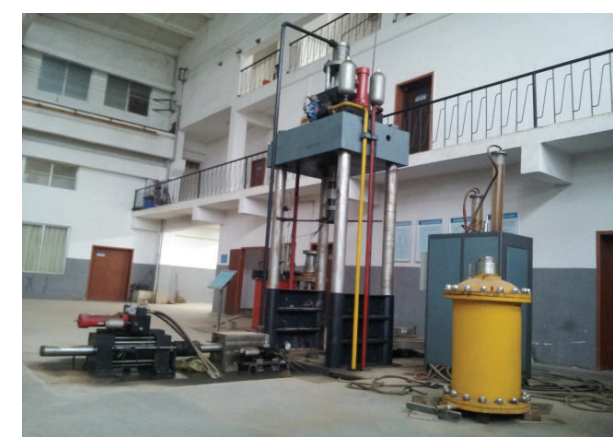

Figure 9: TAJ-2000 large static triaxial test apparatus.

TABLE 3: Elastic modulus model calculation values of S-RM and FSRM.

\begin{tabular}{lccc}
\hline Rock content & \multicolumn{3}{c}{ Elastic modulus model calculation values (MPa) } \\
& $\begin{array}{c}\text { S-RM } \\
\text { (2-layer) }\end{array}$ & $\begin{array}{c}\text { S-RM } \\
\text { (3-layer) }\end{array}$ & $\begin{array}{c}\text { FS-RM } \\
\text { (3-layer) }\end{array}$ \\
\hline $30 \%$ & 4.617 & 4.665 & 17.765 \\
$40 \%$ & 5.904 & 5.924 & 22.507 \\
$50 \%$ & 7.855 & 7.776 & 29.559 \\
$60 \%$ & 10.931 & 10.604 & 40.448 \\
$70 \%$ & 16.506 & 15.136 & 60.428 \\
\hline
\end{tabular}

The samples are prepared as follows. First, soil samples retrieved from the field are dried and sieved, and they could be divided into soil with a depth of $0 \mathrm{~mm}$ to $5 \mathrm{~mm}$ and gravel with a depth of $5 \mathrm{~mm}$ to $60 \mathrm{~mm}$. Then, according to the requirements of volume percentage $C_{\mathrm{R}}$ of gravel in the test, the samples are prepared with saturation water content. In this test, the diameter and height of the samples were 300 and $600 \mathrm{~mm}$, respectively; the samples were fabricated strictly in accordance with geotechnical test procedures. To ensure uniform distribution of the sample particles, the preparation of the compaction samples is divided into five layers, and compaction control is at $95 \%$. According to the rock content, the soil samples and rock are completely mixed. A typical soilrock mixture is taken to measure its natural moisture content. The quality of each layer of soil-rock mixture is calculated according to the maximum dry density of soul samples in the compaction test. The fully mixed soil-rock mixture is placed in a compaction cylinder five times with manual compaction. The quality of each layer is kept at 1/5 of the total mass. The compaction of the layer is continued until the height of each layer's soil after compaction reached $12 \mathrm{~mm}$, which is $1 / 5$ of the total height. The surface of the sample is roughened before adding the soil sample to the next layer to ensure that no weakness plane is present after compaction, which will contribute to improved combination between levels and enhance the integrity of the sample. When the compactions of all five layers are completed, the mold barrels are removed.

A TAJ-2000 large static triaxial test apparatus (see Figure 9) and a permafrost natural environment simulation system (see Figure 10) are used in the National Engineering Laboratory of High-Speed Railway Construction 


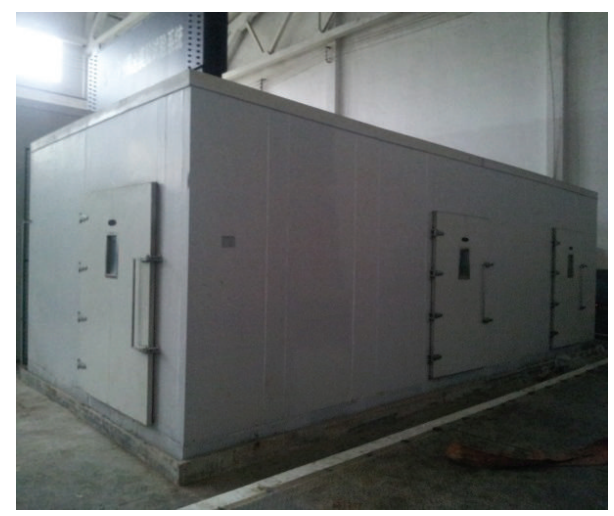

FIGURE 10: Permafrost natural environment simulation system.

TABLE 4: Elastic modulus values of S-RM and FS-RM.

\begin{tabular}{lcc}
\hline Rock content & \multicolumn{2}{c}{$\begin{array}{c}\text { Elastic modulus values by test (MPa) } \\
\text { S-RM (test) }\end{array}$} \\
\hline $30 \%$ & 4.671 & FS-RM (test) \\
$40 \%$ & 5.991 & 23.031 \\
$50 \%$ & 7.631 & 30.021 \\
$60 \%$ & 10.161 & 42.011 \\
$70 \%$ & 15.527 & 63.273 \\
\hline
\end{tabular}

Technology of Central South University as test equipment. The maximum axial stress of the triaxial test apparatus is $2000 \mathrm{kN}$, and the maximum confining pressure is $10 \mathrm{MPa}$. In this study, the sample diameter is $300 \mathrm{~mm}$, and height is $600 \mathrm{~mm}$, and the maximum diameter of the particle is $60 \mathrm{~mm}$. Based on the above test method, the elastic modulus of soil-rock mixture with different rock contents is at the same compaction condition. The test results are shown in Table 4 .

As seen in Table 4, the elastic modulus of soil-rock mixture is significantly higher under the freezing temperature condition than it is under the normal temperature condition. The elastic modulus of soil-rock mixture gradually increases with the increase of the rate of rock content under both the freezing and normal temperature conditions. These findings are consistent with the results based on the micromechanics calculation model, which proves that the calculation model presented in this study is effective.

\subsection{Comparative Analysis between Model Calculation and the} Measured Test. The model calculation values from Model 4.3 are compared with the experimental test values from Model 4.4, and the calculated relative error is presented in Table 5. The comparison between model calculation values and measured test values at normal temperature is shown in Figure 11, and Figure 12 shows the comparison at freezing temperature.

Table 5 shows that the elastic modulus calculated by the micromechanics model of soil-rock mixture is consistent with the experimental values. The maximum relative error is no more than $7.6 \%$. The predicted results for the two-layer

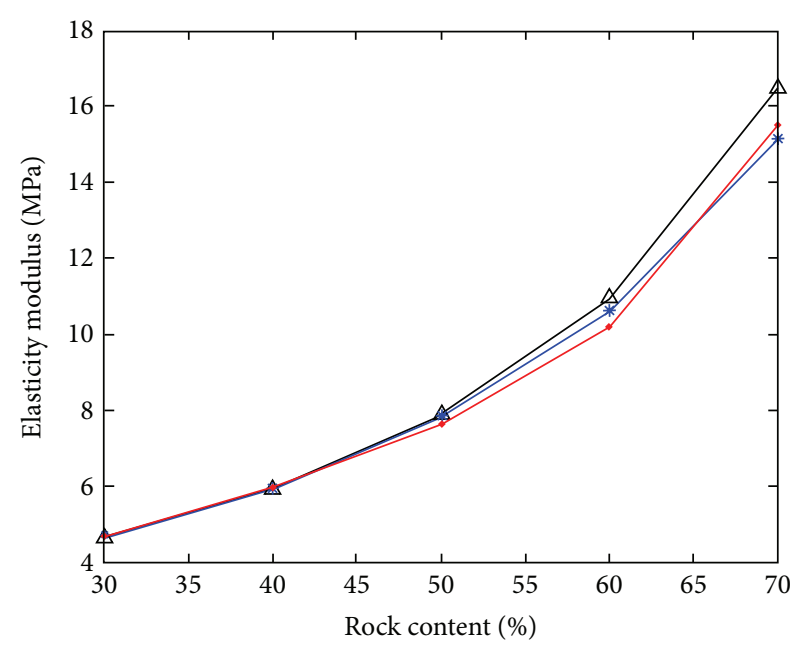

$$
\begin{array}{ll}
\triangle & \text { S-RM (2-layer) } \\
* & \text { S-RM (3-layer) } \\
\rightarrow & \text { S-RM (test) }
\end{array}
$$

FIgURE 11: Comparison between the values calculated by the soilrock mixture model and experimental values at normal temperature condition.

TABLE 5: Relative error chart between model calculation values and the measured test values.

\begin{tabular}{lccc}
\hline \multirow{2}{*}{ Rock content } & \multicolumn{3}{c}{ Relative error (\%) } \\
& S-RM (2-layer) & S-RM (3-layer) & FS-RM (3-layer) \\
\hline $30 \%$ & -1.2 & -0.1 & -1.9 \\
$40 \%$ & -1.5 & -1.1 & -2.3 \\
$50 \%$ & 2.9 & 1.9 & -1.5 \\
$60 \%$ & 7.6 & 4.4 & -3.7 \\
$70 \%$ & 6.3 & -2.5 & -4.5 \\
\hline
\end{tabular}

single inclusion model are good. However, the three-layer double inclusion model is much better than the two-layer single inclusion model in predicting the effect of the soil-rock mixture at normal temperature.

Figures 11 and 12 show that, under the freezing temperature condition, the elastic modulus of soil-rock mixture is significantly greater than that at normal temperature. The elastic modulus calculated by the model at $-20^{\circ} \mathrm{C}$ is compared with the elastic modulus calculated by the model at $20^{\circ} \mathrm{C}$; the average elastic modulus of FS-RM is 3.84 times that of S-RM. The elastic modulus measured at $-20^{\circ} \mathrm{C}$ is compared with the elastic modulus measured results at $20^{\circ} \mathrm{C}$; the average elastic modulus of FS-RM is 3.97 times that of S-RM. At normal or freezing temperature, the elastic modulus of soil-rock mixture increases gradually with the increase in rock content. The elastic modulus of the soilrock mixture increases significantly with the increase in rock content especially when the rock content is between $50 \%$ and $70 \%$.

The error between the values calculated by the model and the measured values can be attributed to the assumed rock shape, size, and distribution form in the model, the 


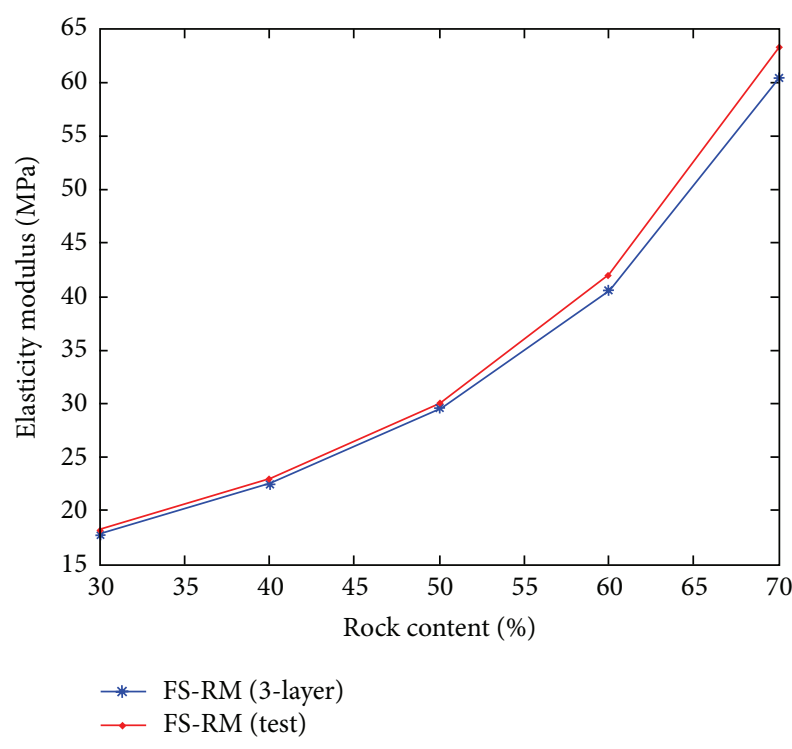

FIGURE 12: Comparison between values calculated by the soil-rock mixture model and experimental values at freezing temperature condition.

differences in internal structure characteristics of the soilrock mixture, and differences in material properties between the substrate, the mixed materials, and the actual component material, among others. However, the relative error is within an acceptable range, which illustrates that the method of the micromechanics model proposed in this study is reasonable and reliable.

\section{Conclusions}

Based on the obtained results, the following conclusions can be made.

(1) This study proposed a two-layer embedded micromechanical model of single inclusion composite material with effective macro performance. This model can predict elastic modulus of the soil-rock mixture under normal temperature condition. Test results show that the predicted results for the two-layer single inclusion model are desirable; however, the three-layer double inclusion model is better than the two-layer single inclusion model in predicting the effect with regard to soil-rock mixture at normal temperature condition.

(2) Based on the multistep homogenization method, this study proposed a micromechanical and threelayer embedded model of double inclusion composite material with effective macro performance. The model can predict the elastic modulus of the soil-rock mixture at freezing temperature condition.

(3) Two micromechanical models are used to calculate the elastic modulus of S-RM and FS-RM with rock contents of $30 \%, 40 \%, 50 \%, 60 \%$, and $70 \%$ to compare with the test results. The maximum relative error between the two methods results is $7.6 \%$, which is within the acceptable range and indicates that the two micromechanical model methods proposed in this study can be used to estimate the mechanical properties of soil-rock mixture under normal or freezing temperature condition.

(4) Under normal or freezing temperature conditions, the elastic modulus of the soil-rock mixture increases gradually with the increase in rock content. The elastic modulus of the soil-rock mixture increases significantly especially when the rock content is between $50 \%$ and $70 \%$.

(5) The elastic modulus of the frozen soil-rock mixture is close to four times higher than that of the soil-rock mixture under normal temperature condition.

\section{Conflict of Interests}

The authors declare that there is no conflict of interests regarding the publication of this study.

\section{Acknowledgments}

The present work was jointly supported by the National Natural Science Foundation of China (Grant no. 500908234), the Major State Basic Research Development Program of China (973 Program, Grant no. 2011CB710604), and the Fundamental Research Funds for the Central Universities of Central South University (Grant no. 72150050525).

\section{References}

[1] W. J. Xu and R. L. Hu, "Conception, classification and significations of soil-rock mixture," Hydrogeology \& Engineering Geology, vol. 36, no. 4, pp. 50-56, 2009.

[2] G. Bourkas, I. Prassianakis, V. Kytopoulos, E. Sideridis, and C. Younis, "Estimation of elastic moduli of particulate composites by new models and comparison with moduli measured by tension, dynamic, and ultrasonic tests," Advances in Materials Science and Engineering, vol. 2010, Article ID 891824, 13 pages, 2010.

[3] Z. Liu, J. Shao, W. Xu, and C. Shi, "Estimation of elasticity of porous rock based on mineral composition and microstructure," Advances in Materials Science and Engineering, vol. 2013, Article ID 512727, 10 pages, 2013.

[4] Y. G. Yang, F. Gao, H. M. Cheng, Y. M. Lai, and X. X. Zhang, "Researches on the constitutive models of artificial frozen silt in underground engineering," Advances in Materials Science and Engineering, vol. 2014, Article ID 902164, 8 pages, 2014.

[5] Z. Zhou, H.-G. Wang, H.-L. Fu, and B.-C. Liu, "Influences of rainfall infiltration on stability of accumulation slope by insitu monitoring test," Journal of Central South University of Technology, vol. 16, no. 2, pp. 297-302, 2009.

[6] E. Medley, Then engineering characterization of mélanges and similar Block-in-Mixtrix Rocks (Bimrocks) [Ph.D. thesis], Department of Civil Engineering, University of California, Berkeley, Calif, USA, 1994.

[7] S. P. Parker, Dictionary of Geology and Mineralogy, McGrawHill, New York, NY, USA, 2003. 
[8] Z. Zhou, Study on the fluid-solid coupling characteristic of soil and rock blending landslide and its prediction and forecast [Ph.D. thesis], Central South University at Changsha, 2006.

[9] Z. Hashin, "Theory of mechanical behavior of heterogeneous media," Applied Mechanics Reviews, vol. 17, no. 1, pp. 1-9, 1964.

[10] C. M. Bishop, M. Tang, R. M. Cannon, and W. C. Carter, "Continuum modelling and representations of interfaces and their transitions in materials," Materials Science and Engineering A, vol. 422, no. 1-2, pp. 102-114, 2006.

[11] H. K. Lee and S. H. Pyo, "Multi-level modeling of effective elastic behavior and progressive weakened interface in particulate composites," Composites Science and Technology, vol. 68, no. 2, pp. 387-397, 2008.

[12] M. Wang and N. Pan, "Predictions of effective physical properties of complex multiphase materials," Materials Science and Engineering R: Reports, vol. 63, no. 1, pp. 1-30, 2008.

[13] C. P. Wong and R. S. Bollampally, "Thermal conductivity, elastic modulus, and coefficient of thermal expansion of polymer composites filled with ceramic particles for electronic packaging," Journal of Applied Polymer Science, vol. 74, no. 14, pp. 33963403, 1999.

[14] H. Ma, M.-Z. Gao, J.-K. Zhang, and Q.-F. Yu, “Theoretical model developed for equivalent elastic modulus estimation of cobblestone-soil matrix," Rock and Soil Mechanics, vol. 32, no. 12, pp. 3642-3646, 2011.

[15] Q. Y. Zhang, "Shear strength and stress-strain properties of sand-gravel materials," Hydro Science and Engineering, vol. 1, no. 3, pp. 133-145, 1985.

[16] P. Jiang and S. B. Yang, "Experimental study on rheologic behavior and long term strength of gravel in Chengdu area," Journal of Geological Hazards and Environment Preservation, vol. 9, no. 1, pp. 38-42, 1998.

[17] B. Jia and R. H. Wang, "Dynamic characteristics of sandy rock soil," Industrial Construction, vol. 36, no. 5, pp. 71-74, 2006.

[18] R. H. Wang, B. Jia, A. F. Deng, and J. Wang, "Dynamic triaxial testing study on dynamic characteristics of sandy rock soil," Chinese Journal of Rock Mechanics and Engineering, vol. 25, supplement 2, pp. 4059-4064, 2006.

[19] S.-L. Qin, S.-X. Chen, Z. Han, and X.-C. Xu, "Large-scale triaxial test study of behavior of over coarse-grained soils," Rock and Soil Mechanics, vol. 31, supplement 2, pp. 189-193, 2010.

[20] G. Q. Li, Y. Q. Li, F. J. B. Metcalf, and S.-S. Pang, "Elastic modulus prediction of asphalt concrete," Journal of Materials in Civil Engineering, vol. 11, no. 3, pp. 236-241, 1999.

[21] S. P. Timoshenko and J. N. Goodier, Theory of Elasticity, McGraw-Hill, New York, NY, USA, 3rd edition, 1970.

[22] R. M. Christensen and K. H. Lo, "Solutions for effective shear properties in three phase sphere and cylinder models," Journal of the Mechanics and Physics of Solids, vol. 27, no. 4, pp. 315-330, 1979.

[23] Z. M. Xiao and B. J. Chen, "On the interaction between an edge dislocation and a coated inclusion," International Journal of Solids and Structures, vol. 38, no. 15, pp. 2533-2548, 2001.

[24] Z. M. Xiao and J. Bai, "On piezoelectric inhomogeneity related problems-part II: a circular piezoelectric inhomogeneity interacting with a nearby crack," International Journal of Engineering Science, vol. 37, no. 8, pp. 961-976, 1999.

[25] Q. S. Yang and X. Tao, "Stepping scheme for multi-inclusion problem," Acta Materiae Compositae Sinica, vol. 24, no. 6, pp. 128-134, 2007.
[26] Q. G. Guo, Engineering Properties of Coarse Grained Soil and Its Application, Yellow River Conservancy Press, Zhengzhou, China, 1998.

[27] M. A. Dafalla, "Effects of clay and moisture content on direct shear tests for clay-sand mixtures," Advances in Materials Science and Engineering, vol. 2013, Article ID 562726, 8 pages, 2013.

[28] Z.-Z. Wang, S.-Y. Mu, Y.-H. Niu, L.-J. Chen, J. Liu, and X.-D. Liu, "Predictions of elastic constants and strength of transverse isotropic frozen soil," Rock and Soil Mechanics, vol. 29, supplement 1, pp. 475-480, 2008. 

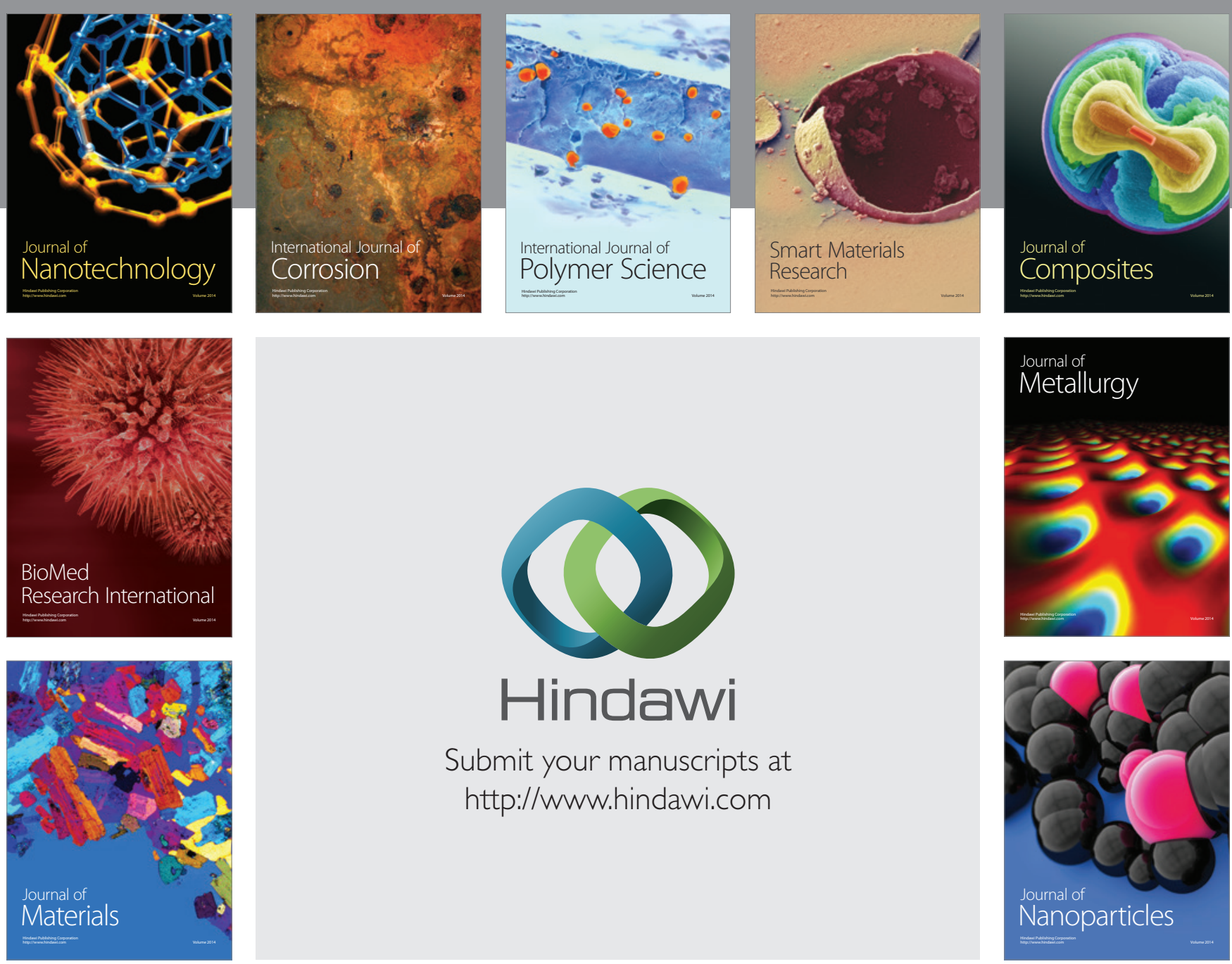

Submit your manuscripts at http://www.hindawi.com
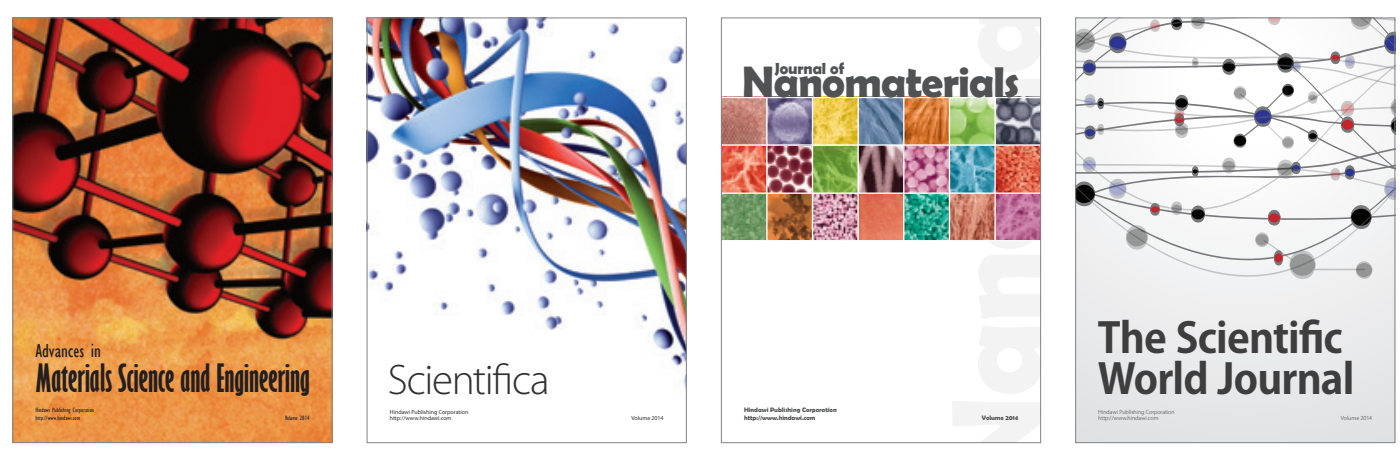

\section{The Scientific World Journal}
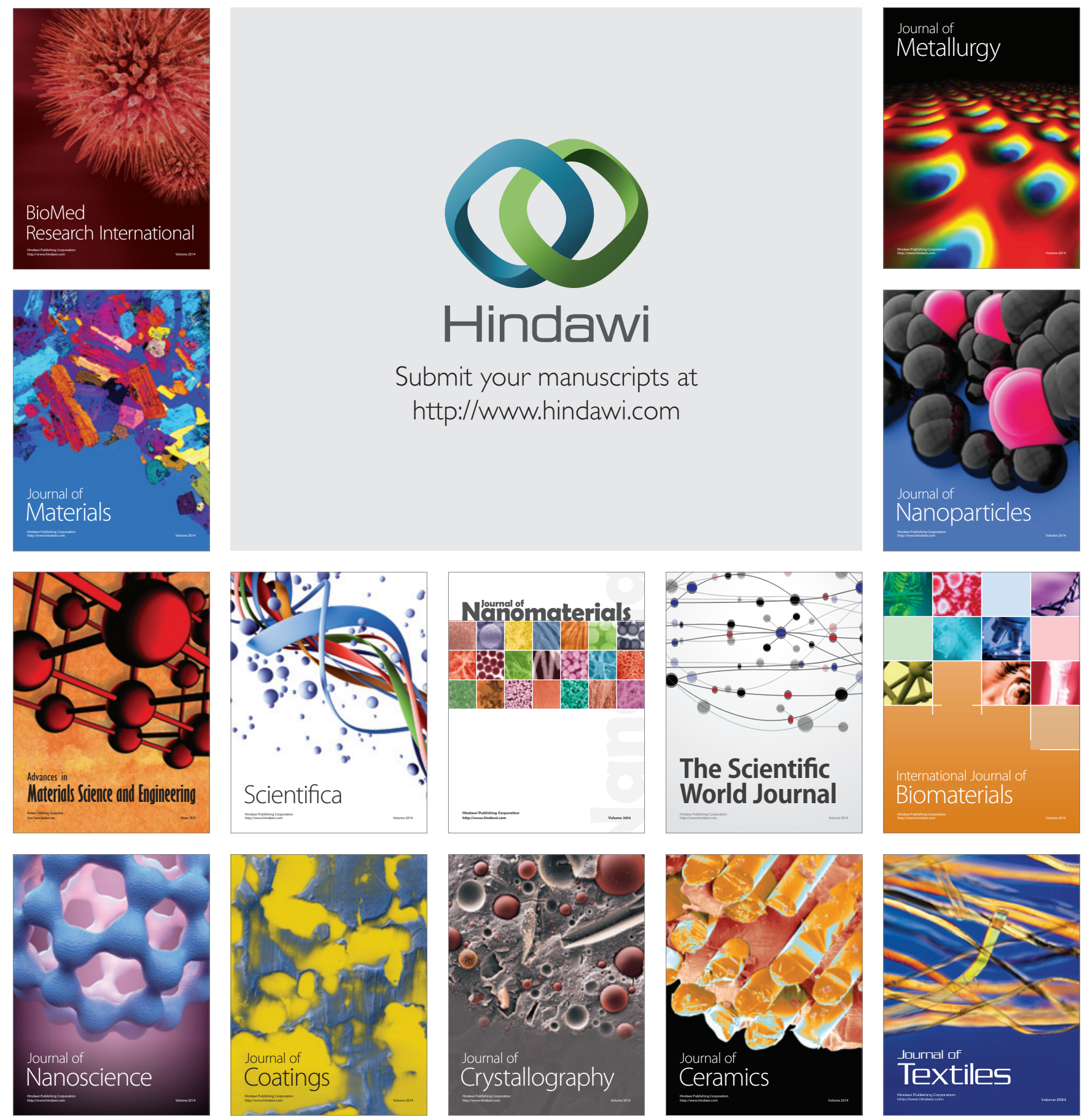